Ban L, Tata LJ, Fiaschi L, Card T.

Limited risks of major congenital anomalies

in children of mothers with IBD and effects of medications.

Gastroenterology.

2014 Jan;146(1):76-84. doi: 10.1053/j.gastro.2013.09.061.

Epub 2013 Oct 12.

PubMed PMID: 24126096.

This is the full text of a work available in its final form at

http://www.gastrojournal.org/article/S0016-5085(13)01437-6/fulltext

(C) 2014. This manuscript version is made available under the CC-BY-NC-ND 4.0 license http://creativecommons.org/licenses/by-nc-nd/4.0/ 


\section{Limited Risks of Major Congenital Anomalies in Children of Mothers with IBD and Effects of Medications.}

Short Title: Major congenital anomalies and IBD

Lu Ban ${ }^{1}$, Laila Jal Tata ${ }^{1}$, Linda Fiaschi ${ }^{1}$ and Timothy Card $^{1,2}$

1. Division of Epidemiology \& Public Health, University of Nottingham, Nottingham, United Kingdom

2. Nottingham Digestive Diseases Centre, National Institute for Health Research Biomedical

Research Unit, Queen's Medical Centre, Nottingham University Hospitals National Health Service

Trust, Nottingham, UK

Grant Support This study was funded by the National Association for Colitis and Crohn's Disease.

The funders had no role in the conception, conduct, interpretation or writing up of the study,

Abbreviations IBD=inflammatory bowel disease; $C D=C$ rohn's disease; $U C=$ =ulcerative colitis; $\mathrm{AOR}=$ adjusted odds ratio; $95 \% \mathrm{Cl}=95 \%$ confidence interval; $\mathrm{MCA}=$ major congenital anomaly; 5 ASA $=5$-aminosalicylic acid

Correspondence Timothy Card (tim.card@nottingham.ac.uk)

Division of Epidemiology \& Public Health, Clinical Sciences Building, Nottingham City Hospital, Hucknall Road, Nottingham NG5 1PB; tel +44(0)115 8231346

Disclosures No conflict of Interests was declared.

Author Contributions All authors contributed to conception and design of the study, analysis and interpretation of the study results. TC, LJT and LB drafted the manuscript. All authors revised the manuscript. TC supervised the study and is guarantor. 


\section{Abstract}

Background \& Aims: Concerns persist about the risk of major congenital anomalies (MCA) in children of women with inflammatory bowel disease (IBD), and whether medication use affects risk. We assessed these risks, and variations in use of medications by women with IBD before, during, and after pregnancy.

Methods: We accessed data on children born to women 15-45 y old from 1990 through 2010 using a mother-child linked dataset from an electronic database of primary care records containing medical diagnoses, events, and drug prescriptions from across the United Kingdom. We identified pregnant women with IBD and all prescriptions for 5-aminosalicylates, azathioprine/6-mercaptopurine, and corticosteroids were extracted from their primary care records. We calculated risks of MCA in children of mothers with and without IBD, and in children exposed or not exposed to 5-aminosalicylates, azathioprine/6-mercaptopurine, or corticosteroids during their first trimester of fetal development. Logistic regression with a generalized estimating equation was used to provide risk estimates adjusted for confounders. We calculated proportions of women taking medications before, during, and after pregnancy and assessed whether cessation was associated with subsequent disease flares.

Results: Risks of MCA in 1703 children of mothers with and 384,811 children of mothers without IBD were $2.7 \%$ and $2.8 \%$ respectively. This corresponded to an adjusted odds ratio of 0.98 (95\% confidence interval $[\mathrm{CI}], 0.73-1.31)$. In children of women with IBD, the adjusted odds ratios of MCA associated with drug use were $0.82(95 \% \mathrm{Cl}, 0.42-1.61)$ for 5 -aminosalicylates, $0.48(95 \% \mathrm{Cl}, 0.15-$ 1.50) for corticosteroids, and $1.27(95 \% \mathrm{Cl}, 0.48-3.39)$ for azathioprine/6-mercaptopurine. No increases in heart, limb, or genital anomalies were found in children of women with IBD; $31.2 \%$ of women discontinued 5-aminosalicylates and $24.6 \%$ discontinued azathioprine/6-mercaptopurine in early pregnancy. The risk of flares later in pregnancy was not related to cessation of medication.

Conclusions: We found no evidence that IBD during pregnancy or medical therapy for IBD during pregnancy increases the risk of MCA in children. Patients should receive appropriate guidance on use of medication before and during pregnancy.

KEYWORDS: Crohn's disease; ulcerative colitis; birth defects; treatment 


\section{Introduction}

Inflammatory bowel disease (IBD) commonly affects women of reproductive age ${ }^{1}$, and is often managed using drugs recognised to have potential for toxicity in adults ${ }^{2,3}$. Since it is unethical to test the safety of almost all drugs in pregnancy in clinical trials, the potential for teratogenicity of drugs taken during pregnancy has been a pressing and natural concern of all pregnant women or those attempting to become so. This particularly affects women with chronic medical conditions that may only be effectively managed with medication. The risks of adverse effects in their children associated with the drugs taken during pregnancy continue to be a concern for women with IBD which has led to a number of studies in recent years ${ }^{4-7}$. However, IBD is a relatively uncommon condition and coupled with the rarity of individual congenital anomalies and mostly retrospectively collected data available to researchers, it remains unclear whether there is an overall increase in congenital anomalies among children born to women with IBD, and if so whether this is driven by medications. A meta-analysis published in 2007 showed that maternal IBD in pregnancy may be associated with an over 2-fold increased risk of congenital anomalies, which was more evident in women with ulcerative colitis (UC) than in women with Crohn's disease (CD), however only 637 women with IBD across four studies were included ${ }^{8}$. A more recent population-based study assessing risks only in women with UC combined Danish and Swedish registry data and authors found no increased risk of congenital anomalies ${ }^{9}$. Both the meta-analysis and the later study, however, did not examine the effect of drug treatment separated from the effect of disease, nor the risks of system-specific congenital anomalies. In addition, the extent to which published studies include minor congenital anomalies is not clear, and their inclusion could increase the likelihood of ascertainment bias, thus inflating the risk of congenital anomalies in children born to mothers with IBD.

The gestational safety of a number of commonly used drugs for IBD has been assessed and studies have not shown large or consistent increases in the occurrence of congenital anomalies ${ }^{10-13}$, but have nonetheless been unable to completely satisfy many of the safety of these drugs in pregnancy, particularly due to the small number of exposures included. Based on this evidence as well as the known tendency of IBD to flare upon cessation of disease suppressing medication and the recognised 
excess of other adverse pregnancy outcomes which may be driven by disease activity ${ }^{14}$, a number of guidelines have been writte ${ }^{15-19}$. These propose that women should not cease drug treatment that is maintaining their IBD in remission for the purpose of becoming pregnant or protecting a pregnancy, yet very recent studies indicate that women remain fearful of the potential harm of their drugs during pregnancy $^{20,21}$.

In view of the lack of certainty as to the risk of congenital anomalies in children born to women with IBD and among those taking suppressive medications, we have conducted a very large cohort study of pregnancies using United Kingdom (UK) primary care data. We set out to examine the hypotheses that maternal IBD per se is associated with an increased risk of major congenital anomaly and that corticosteroid, 5-aminosalicylic acid (5-ASA) and Azathioprine/6-Mercaptopurine use is associated with increased risks among women with IBD. Given the current lack of knowledge about how women and clinicians respond to perceived risks (in terms of drug use in pregnancy) despite existing guidelines, we also assessed prescribing patterns over a year before pregnancy, during and after pregnancy in detail and the extent to which cessation of treatment when pregnant was related to subsequent IBD flares in pregnancy. 


\section{Methods}

\section{Defining the study population}

We studied all live singleton children born to women aged 15-45 years between 1990 and 2010 using an anonymised mother-child-linked dataset from The Health Improvement Network (THIN), a nationally representative UK database of primary care records containing medical diagnoses, events and drug prescriptions ${ }^{22}$. For each mother-child pair, we defined women as having IBD in pregnancy if they had a diagnosis of IBD ever before childbirth or if they had a diagnosis after childbirth but prescriptions of 5-ASA at some point before childbirth. Mothers with IBD were classified as having CD if they had diagnoses specifying this condition regardless of whether they also had diagnoses of UC, as having UC if they had recordings of UC but not CD, and as unclassified if their coding for IBD did not specify CD or UC. Among mothers with IBD, all prescriptions for 5-ASA, azathioprine/6Mercaptopurine or corticosteroids (including both oral and rectal corticosteroids) were extracted from their primary care electronic health records. Exposure in the first trimester was considered to occur if a prescription was issued between four weeks before a woman's estimated conception date and 12 weeks after. Beginning this period four weeks before conception enabled inclusion of drug prescriptions received immediately before pregnancy and potentially used in early pregnancy.

\section{Defining major and system-specific congenital anomalies}

All diagnoses of major congenital anomalies were identified in the children's medical records using Read codes, classified into system-specific groups according to the European Surveillance of Congenital Malformations (EUROCAT) subgroups ${ }^{23}$. These subgroups are defined using International Classification of Diseases (ICD-10) codes to which our coding was mapped. Children with records of genetic anomalies attributed to known teratogens (such as Read codes for anomalies due to maternal infections and foetal alcohol syndrome) were excluded, comprising less than $0.1 \%$ of the original child population.

\section{Defining maternal co-variables}

We extracted data on the following maternal characteristics: maternal age at childbirth, calendar year of childbirth (categorised as 1990-1996, 1997-2003 and 2004-2010), maternal smoking status and 
socioeconomic deprivation measured using the Townsend Index of Deprivation, in quintiles ${ }^{24}$.

Maternal smoking status was defined using women's most recent smoking status before childbirth, categorised as non-smoker, current smoker, ex-smoker and unknown.

\section{Statistical analyses}

We calculated absolute risks (per 10,000 live births) of all major congenital anomalies and anomalies in 14 system-specific groups for children born to women without IBD, women with any type of IBD and women with CD or UC separately. Since there were only 104 women with unclassified IBD, we did not separately assess this group. Logistic regression was used to estimate odds ratios (ORs) with $95 \%$ confidence intervals $(95 \% \mathrm{Cls})$ for the association of any major congenital anomaly and the three most common system-specific subgroups (heart, limb and genital anomalies) in children born to mothers with IBD compared to those born to mothers without IBD who acted as controls, with full adjustment for co-variables.

Among women with IBD, we calculated the proportions of children exposed to 5-ASA, corticosteroids or Azathioprine/6-Mercaptopurine in the first trimester of pregnancy and used logistic regression to assess the association of each type of antenatal drug exposure with the risks of major congenital anomalies. Because prescriptions for Azathioprine and 6-Mercaptopurine are commonly for long periods, we additionally conducted a sensitivity analysis using an extended antenatal exposure window beginning at 12 (instead of four) weeks before conception. For all regression analyses the generalised estimating equation approach with exchangeable correlation structure was applied to control for potential clustering effects when children were born to the same woman in consecutive pregnancies.

To examine the extent to which women and physicians may modify IBD drug treatment in relation to pregnancy, we calculated the proportions of children exposed to Azathioprine/6-Mercaptopurine in each trimester of pregnancy and in 3-month periods extending to 15 months before and after pregnancy. We also assessed whether women who were on drug treatment in the 9 months before pregnancy continued or discontinued drug treatment in the first trimester of pregnancy and the occurrence of flares (defined using prescriptions of oral and rectal corticosteroids) in the second and third trimesters of pregnancy and also in the 9 months postpartum. We additionally subdivided the 
analysis by disease type and presented the results for UC and CD separately. All analyses were carried out using Stata SE 11.0 (Stata Corp., TX, USA).

Ethical approval for this research was obtained from the South-East Multicentre Research Ethics Committee (SE-REC) reference 04/MRE01/9. 


\section{Results}

Of 386,514 children, $0.4 \%$ were born to mothers who had IBD before childbirth $(n=1,703)$ and $0.2 \%$ to mothers with $C D$ ( $n=893)$. Table 1 shows maternal characteristics for children born to women with and without IBD. Compared with children in the control group, children in the IBD group were less likely to be from socioeconomically deprived areas, were more likely to be born later in the study period and their mothers were slightly older (Table 1). However, children born to women with CD were more likely to be from socioeconomically deprived areas and their mothers were more likely to be current smokers compared with children born to women with UC (Table 1).

\section{Absolute and relative risks of major congenital anomalies with IBD}

Tables 2 and 3 show the absolute and relative risks of any major congenital anomaly and systemspecific anomalies in children born to women with IBD compared with children in the control group. The overall prevalence of any major congenital anomaly was $2.7 \%$ in children born to women with IBD, $3.7 \%$ in children born to women with UC, $1.9 \%$ in children born to women with $\mathrm{CD}$, and $2.8 \%$ in in the control group (adjusted ORs=0.98 [95\% $\mathrm{Cl} 0.73-1.31]$ for IBD overall, 1.34 [95\% $\mathrm{Cl} 0.91-1.97]$ for UC, and $0.68[95 \% \mathrm{Cl} 0.41-1.13]$ for $\mathrm{CD}$ ). For system-specific anomalies, there were variations in absolute risks across groups with no consistent increases or decreases (Table 2). All 95\%Cls of ORs included unity (Table 3).

\section{Risks of major congenital anomalies with drug treatment for IBD}

The proportions of children exposed to 5-ASA in the first trimester were $32.4 \%$ in children born to women with IBD, $40.9 \%$ in children born to women with UC, and $28.6 \%$ for CD (Table 4). Exposure to corticosteroids was similar for women with $U C$ and $C D$ whereas women with $C D$ were more likely to be exposed to Azathioprine/6-Mercaptopurine in early pregnancy than women with UC (Table 4). Table 5 shows the relative risks of any major congenital anomaly and system-specific groups (heart, limb and genital system) in children exposed to 5-ASA, corticosteroids or Azathioprine/6Mercaptopurine in the first trimester of pregnancy compared with children born to women with IBD but not prescribed the respective drugs. All 95\% Cls of adjusted ORs included unity (Table 5). The ORs for Azathioprine/6-Mercaptopurine were similar in the sensitivity analysis which included prescriptions 
12 weeks before to 12 weeks after conception (adjusted $\mathrm{OR}=1.50,95 \% \mathrm{Cl}$ 0.79-2.84 for any major congenital anomaly).

\section{Changes in preconception, antenatal and postnatal medication use}

Figure 1 shows that in 1,162 women with IBD who were registered with their GP throughout the 15 months before pregnancy, during pregnancy and in 15 months postpartum, the proportions of women prescribed Azathioprine/6-Mercaptopurine decreased shortly before conception and generally continued decreasing throughout pregnancy, particularly for women with CD. In the post-partum period, prescribing prevalence rose again to a similar level to that in the months before conception (Figure 1).

Going on to assess the occurrence of flares of disease in relation to changes in drug use, we found that in 487 women who were prescribed 5-ASA in the 9 months before pregnancy (Figure 2a), 68.8\% (335) of them had another prescription in the first trimester of pregnancy, of whom 56 (16.7\%) had a flare later in pregnancy and $57(17.0 \%)$ had a flare postpartum; however in the remaining 152 women who discontinued their medication in early pregnancy $18.4 \%$ had a flare later in pregnancy and $11.2 \%$ had a flare postpartum. Among 122 women prescribed Azathioprine/6-Mercaptopurine in the 9 months before pregnancy (Figure 2a), 75.4\% (92) of them continued on medication when pregnant, of whom $8.7 \%$ and $13.0 \%$ developed a subsequent flare in later pregnancy and postpartum respectively. Of the 30 women who discontinued Azathioprine/6-Mercaptopurine use in pregnancy $10.0 \%$ had a flare later in pregnancy and another $10.0 \%$ had a flare in the 9 -month postpartum period. For neither drug was the proportion of women experiencing a flare significantly different between those ceasing and continuing the drug $(p=0.65$ and 0.17 for 5 -ASA discontinuation and $p=0.83$ and 0.93 for Azathioprine/6-Mercaptopurine discontinuation with flares in later pregnancy and postpartum respectively). Similar figures subdivided between Crohn's disease and UC are given in figures $2 \mathrm{~b}$ and $2 \mathrm{c}$ demonstrating a higher overall rate of flare in UC. Due to inadequate power statistical testing of the effect of drug cessation on flares was not conducted in the subdivided analysis. 


\section{Discussion}

\section{Principal findings}

This study is among the largest in terms of numbers of women with IBD included, numbers of births and numbers of major congenital anomalies which has yet been conducted to assess risks of congenital anomalies among the children of women with IBD. It has demonstrated no overall excess risk of major congenital anomaly in children born to mothers with IBD and none associated with Azathioprine/6-Mercaptopurine, corticosteroids or 5-ASA use within the maternal IBD population. The study has also been able to describe how women vary their use of medication for IBD around the time of pregnancy in order to assess not only whether they are stopping 5-ASA and Azathioprine/6Mercaptopurine, but also whether doing so is associated with increased use of corticosteroids (as a proxy for flares of IBD) during pregnancy. We have found that an appreciable proportion (between 25 and $31 \%$ ) of women stop receiving prescriptions, but this was not associated with a significant increase in flares later in pregnancy.

\section{Strengths and limitations}

Besides its size and consequent precision, our study has a number of other advantages. As the selection of both cases and controls is truly population based, and the ascertainment of outcomes is independent of a pre-designed research protocol (since we are using routinely collected data), we have minimised the opportunities for selection and ascertainment bias beyond that which occurs routinely in clinical practice. In addition, the availability of contemporaneously gathered prescription data provides us the opportunity to examine drug effects, and data on maternal smoking, socioeconomic status and maternal age allow us to correct for some important potential confounders. Our methodology of course also has limitations. Though our study is large, due to the rarity of outcomes, the power is still limited. We are for example arguably underpowered to compare the risks of congenital anomalies between UC and Crohn's and are underpowered to investigate most individual anomalies. Also since we have used anonymised routinely collected records, we have been unable to go back to the patients or their paper records to validate the information we have used. As regards our exposure data, the diagnosis of IBD has been previously validated in UK electronic 
general practice records and found to be highly reliable ${ }^{25}$, and prescriptions are automatically entered at the point of issue on the database such that error in their measurement will be minimal. The diagnosis of our congenital anomaly outcome in electronic primary care data has also been validated against written primary care records ${ }^{26}$. Furthermore, prevalence estimates across all system-specific groups and for specific major congenital anomaly diagnoses are comparable to those reported in UK registers of the European Surveillance of Congenital Anomalies network ${ }^{27}$ and in the case of trisomy 21 the expected variation by age is shown ${ }^{28}$. Our inability to individually check children however might still permit a bias to arise if, for example, the children born to women with IBD are more carefully assessed. This bias would of course be one to which essentially all previous studies have been susceptible, and would bias towards finding an association with congenital abnormalities which we did not detect. Unlike most previous studies in this field ${ }^{7,10,29-33}$ we have tried to minimise ascertainment bias of this type by limiting our analysis to major congenital anomalies which are unlikely to go undetected in the offspring of healthy mothers.

\section{Interpretation in the context of previous literature}

Recent US data from a large prospective study as yet reported in abstract only (the PIANO study) are in general in keeping with our findings that thiopurines are not associated with increased congenital anomalies ${ }^{34}$. The previous literature on congenital anomalies and IBD is however plagued by studies with limited power. Though a meta-analysis was conducted in 2007 to attempt to overcome this and found an excess of congenital anomalies in children born to women with IBD (OR=2.37, $95 \% \mathrm{Cl} 1.47$ 3.82), more evident in UC $(\mathrm{OR}=3.88,95 \% \mathrm{Cl} 1.41-10.67)$ than in $\mathrm{CD}(\mathrm{OR}=2.14,95 \% \mathrm{Cl} 0.97-4.74)^{8}$, it included only five studies with 702 pregnancies in total, which reduced to only 539 once the three smallest studies ${ }^{30,33,35}(<100$ pregnancies in women with IBD) had been excluded. The estimates from the meta-analysis are therefore driven by three studies ${ }^{29,35,36}$, but these among them studied only about one third of the pregnancies exposed to IBD presented in our current paper and half the number reported in the PIANO study . Although a large population-based study published in 2011 using both Danish and Swedish registry data ${ }^{9}$ included 84 congenital anomalies in children born to women with UC and found no increased risk of congenital anomalies (OR=1.05, 95\% $\mathrm{Cl}$ 0.84-1.31), 
this later study compared only UC patients with all other non-UC patients and did not assess potential drug exposures or diagnoses of $C D^{9}$. When considering the difference between our results and these previous studies it is also important to note that like our study they were reliant upon clinical databases for their information, and though the Danish and Swedish study excluded some minor congenital anomalies, they did not limit analysis to major anomalies alone and so may have been more vulnerable to bias in the ascertainment of anomalies than in the current study.

Perhaps more important than the risk of congenital anomalies from merely having IBD, which women cannot prevent, is the risk related to drug exposures which they may be able to alter. In this regard our results are broadly consistent with the previous literature ${ }^{11-13}$, showing no excess risk related to the use of 5-ASA, corticosteroids or Azathioprine/6-Mercaptopurine. Of course once we begin studying small subgroups such as that of women treated in pregnancy with specific drugs, the power of our study is greatly reduced. This somewhat limits the comfort provided by for example our estimate of a non-significant reduction in congenital anomalies in children born to mothers who took 5-ASA during pregnancy $(\mathrm{OR}=0.82,95 \% \mathrm{Cl} 0.42-1.61)$. The idea that no significant excess risk from the use of these drugs exists, is however supported by a 2008 meta-analysis which found no statistically significant excess of congenital anomalies and a point estimate of a 1.16-fold excess risk for 5-ASA exposure ${ }^{11}$. A similar situation exists for Azathioprine/6-Mercaptopurine, in which a recent meta-analysis on the effects of thiopurines on birth outcomes in patients with IBD found a nonsignificant excess of risk of congenital anomalies and an OR of $1.45(95 \% \mathrm{Cl} 0.99-2.13)^{13}$. The situation is slightly different for corticosteroids where the most recent available meta-analysis, though it does not show an overall risk of any MCA, does suggest an excess of cleft lip and palate ${ }^{12}$ which we lack power to assess even to the extent which we have examined the overall risk.

Based upon the existing literature there is a near uniformity of recommendations on the use of IBD medications in pregnancy ${ }^{15,19}$. Though it is recommended that methotrexate should not be used in pregnancy $^{37}$, for 5-ASA, Azathioprine/6-Mercaptopurine and short term administration of corticosteroids, the risks of disease if their use is prevented is thought to outweigh the risks to the mother and foetus of continuing the medication ${ }^{15}$. How well this message has been communicated to 
patients, and to what extent the advice is acted upon has however been little studied. Previous research conducted in Australia showed that women with IBD were fearful of the effects of medication upon pregnancy with $30 \%$ specifically fearing congenital anomalies ${ }^{38}$ and as many as $84 \%$ fearing some adverse effect upon the pregnancy ${ }^{14}$. We also have data from Denmark where studies in UC and CD have demonstrated that not only do women with IBD fear the effects of their medications, but this also leads to reduced adherence ${ }^{20,21}$. Since all of these studies were based upon questionnaires, and therefore are susceptible both to recall and response biases, the data they provided on medication usage by women with IBD during pregnancy may be less reliable than the prospectively recorded prescription data which we have analysed despite the fact that we are unable to assess compliance. These studies are also unable to help us judge to what extent non-compliance with medication during pregnancy may have been associated with changes in disease activity. The current study, though it can tell us nothing of the reasons for women's choices or of their fears, can help fill in some of these gaps. We have shown that about 3/4 of women taking 5-ASA or Azathioprine/6Mercaptopurine prior to pregnancy continue to do so during pregnancy, but that about $1 / 4$ cease to receive prescriptions (and therefore presumably stop). If we accept the use of corticosteroid prescriptions as a proxy for disease activity (as previously validated in UK primary care data by Lewis et $\left.a f^{39}\right)$, then the analysis we present in Figure 2 shows only a slight non-significant increase in the proportion of women who have flares following the cessation of 5-ASA and Azathioprine/6Mercaptopurine in early pregnancy. When interpreting this finding though it may be instructive to consider the likely scenario in which medications are stopped. Women will be less likely to stop their medication if they have symptomatic (even mildly symptomatic) disease, and so it is likely that those who stopped were at the time of cessation on average suffering less from disease activity than those who continued taking medication. In this context a small albeit not statistically significant increase in flares in those stopping 5-ASA or Azathioprine/6-Mercaptopurine may well signal that the effect of cessation in pregnancy is the same as that outside it, and promotes increased flares ${ }^{40}$.

\section{Conclusion}


We conclude that neither IBD, nor its common medical treatments are likely to be major risk factors for congenital anomalies, but that this message may not have reached all pregnant women. About one quarter of women stop their medication in pregnancy. Though this may increase the risk of flares later in pregnancy, we were unable to demonstrate such increase. 


\section{Reference}

1. Binder V. Epidemiology of IBD during the twentieth century: an integrated view. Best Practice \& Research Clinical Gastroenterology 2004;18:463-479.

2. Zorzi F, Zuzzi S, Onali S, et al. Efficacy and safety of infliximab and adalimumab in Crohn's disease: a single centre study. Alimentary Pharmacology \& Therapeutics 2012;35:1397-1407.

3. Weaver JL. Establishing the carcinogenic risk of immunomodulatory drugs. Toxicol Pathol 2012;40:267-271.

4. Bortoli A, Pedersen N, Duricova D, et al. Pregnancy outcome in inflammatory bowel disease: prospective European case-control ECCO-EpiCom study, 2003-2006. Alimentary Pharmacology \& Therapeutics 2011;34:724-734.

5. Baird DD, Narendranathan M, Sandler RS. Increased risk of preterm birth for women with inflammatory bowel disease. Gastroenterology 1990;99:987-994.

6. Bush M, Patel S, Lapinski R, et al. Perinatal outcomes in inflammatory bowel disease. Journal of Maternal-Fetal and Neonatal Medicine 2004;15:237-241.

7. Dominitz JA, Young JC, Boyko EJ. Outcomes of infants born to mothers with inflammatory bowel disease: a population-based cohort study. Am J Gastroenterol 2002;97:641-8.

8. Cornish J, Tan E, Teare J, et al. A meta-analysis on the influence of inflammatory bowel disease on pregnancy. Gut 2007;56:830-7.

9. Stephansson $\mathrm{O}$, Larsson $\mathrm{H}$, Pedersen $\mathrm{L}$, et al. Congenital abnormalities and other birth outcomes in children born to women with ulcerative colitis in Denmark and Sweden. Inflammatory Bowel Diseases 2011;17:795-801.

10. Nørgård B, Pedersen L, Fonager K, et al. Azathioprine, mercaptopurine and birth outcome: a population-based cohort study. Alimentary Pharmacology \& Therapeutics 2003;17:827-834. 
11. Rahimi R, Nikfar S, Rezaie A, et al. Pregnancy outcome in women with inflammatory bowel disease following exposure to 5-aminosalicylic acid drugs: a meta-analysis. Reproductive toxicology (Elmsford, N.Y.) 2008;25:271-5.

12. Park-Wyllie L, Mazzotta $P$, Pastuszak A, et al. Birth defects after maternal exposure to corticosteroids: prospective cohort study and meta-analysis of epidemiological studies. Teratology 2000;62:385-92.

13. Akbari M, Shah S, Velayos FS, et al. Systematic Review and Meta-analysis on the Effects of Thiopurines on Birth Outcomes from Female and Male Patients with Inflammatory Bowel Disease. Inflammatory bowel diseases 2013;19:15-22.

14. Mountifield RE, Prosser R, Bampton $P$, et al. Pregnancy and IBD treatment: this challenging interplay from a patients' perspective. Journal of Crohn's \& colitis 2010;4:176-82.

15. Mowat C, Cole A, Windsor A, et al. Guidelines for the management of inflammatory bowel disease in adults. Gut 2011;60:571-607.

16. Faculty of Sexual and Reproductive Healthcare. Sexual and reproductive health for individuals with inflammatory bowel disease. 2009. Available at: http://www.acpgbi.org.uk/members/guidelines/documents/sexual-and-reproductive-health-forindividuals-with-inflammatory-bowel-disease/ [Accessed January 15, 2013].

17. Dignass A, Lindsay JO, Sturm A, et al. Second European evidence-based Consensus on the diagnosis and management of ulcerative colitis Part 2: Current management. Journal of Crohn's and Colitis 2012;6:991-1030.

18. Dignass A, Assche G Van, Lindsay JO, et al. The second European evidence-based consensus on the diagnosis and management of Crohn's disease: Current management. Journal of Crohn's and Colitis 2010;4:28-62. 
19. Assche G Van, Dignass A, Reinisch W, et al. The second European evidence-based Consensus on the diagnosis and management of Crohn's disease: Special situations. Journal of Crohn's and Colitis 2010;4:63-101.

20. Nielsen MJ, Nørgaard M, Holland-Fisher $\mathrm{P}$, et al. Self-reported antenatal adherence to medical treatment among pregnant women with Crohn's disease. Alimentary pharmacology \& therapeutics 2010;32:49-58.

21. Julsgaard M, Nørgaard M, Hvas CL, et al. Self-reported adherence to medical treatment prior to and during pregnancy among women with ulcerative colitis. Inflammatory bowel diseases $2011 ; 17: 1573-80$.

22. Lewis JD, Schinnar R, Bilker WB, et al. Validation studies of the health improvement network (THIN) database for pharmacoepidemiology research. Pharmacoepidemiology and Drug Safety 2007;16:393-401.

23. EUROCAT. Coding of EUROCAT subgroups of congenital anomalies. 2012. Available at: http://www.eurocat-network.eu/content/EUROCAT-Guide-1.3-Chapter-3.3-Jan13.pdf [Accessed March 22, 2013].

24. Townsend P, Phillimore P, Beattie A. Health and deprivation: Inequality and the North. Croom Helm; 1988.

25. Lewis JD, Brensinger C, Bilker WB, et al. Validity and completeness of the General Practice Research Database for studies of inflammatory bowel disease. Pharmacoepidemiology and drug safety 2002;11:211-8.

26. Charlton MRA, Weil JG, Cunnington MC, et al. Identifying major congenital malformations in the UK General Practice Research Database (GPRD). Drug-Safety 2010;33:741-750.

27. Sokal R, Fleming KM, Tata LJ. Potential of general practice data for congenital anomaly research: Comparison with registry data in the united kingdom: Congenital Anomalies Within 
General Practice Data. Birth Defects Research Part A: Clinical and Molecular Teratology 2013;97:546-553.

28. Tata LJ, Lewis S a, McKeever TM, et al. Effect of maternal asthma, exacerbations and asthma medication use on congenital malformations in offspring: a UK population-based study. Thorax 2008;63:981-7.

29. Elbaz G, Fich A, Levy A, et al. Inflammatory bowel disease and preterm delivery. Int J Gynaecol Obstet 2005;90:193-197.

30. Moser MAJ, Okun NB, Mayes DC, et al. Crohn's disease, pregnancy, and birth weight. The American Journal of Gastroenterology 2000;95:1021-1026.

31. Riis L, Vind I, Politi P, et al. Does pregnancy change the disease course? A study in a European cohort of patients with inflammatory bowel disease. The American Journal of Gastroenterology 2006;101:1539-1545.

32. Nørgård B, Fonager K, Pedersen L, et al. Birth outcome in women exposed to 5-aminosalicylic acid during pregnancy: a Danish cohort study. Gut 2003;52:243-247.

33. Fedorkow DM, Persaud D, Nimrod CA. Inflammatory bowel disease: a controlled study of late pregnancy outcome. Am. J. Obstet. Gynecol. 1989;160:998-1001.

34. Mahadevan U, Martin CF, Sandler RS, et al. PIANO: a 1000 patient prospective registry of pregnancy outcomes in women with IBD exposed to immunomodulators and biologic therapy. Gastroenterology 2012;142:S-149-S-149.

35. Larzilliere I, Beau P. [Chronic inflammatory bowel disease and pregnancy. Case control study]. Gastroenterol. Clin. Biol. 1998;22:1056-1060.

36. Dominitz JA, Young JC, Boyko EJ. Outcomes of infants born to mothers with inflammatory bowel disease: a population-based cohort study. Am J Gastroenterol 2002;97:641-648. 
37. Royal Pharmaceutical Society. Martindale: the complete drug reference. 37th ed. (Sweetman SC, ed.). Pharmaceutical Press; 2011.

38. Mountifield R, Bampton P, Prosser R, et al. Fear and fertility in inflammatory bowel disease: a mismatch of perception and reality affects family planning decisions. Inflammatory Bowel Diseases 2009;15:720-725.

39. Lewis JD, Aberra FN, Lichtenstein GR, et al. Seasonal variation in flares of inflammatory bowel disease. Gastroenterology 2004;126:665-673.

40. Prefontaine E, Sutherland LR, MacDonald JK, et al. Azathioprine or 6-mercaptopurine for maintenance of remission in Crohn's disease. Cochrane Database of Systematic Reviews 2009. Available at: http://onlinelibrary.wiley.com/doi/10.1002/14651858.CD000067.pub2/abstract [Accessed April 5, 2013]. 
Table 1 Characteristics of pregnancies of women with and without IBD before childbirth $(N=386,514)$

\begin{tabular}{|c|c|c|c|c|c|c|c|c|}
\hline & \multirow{2}{*}{\multicolumn{2}{|c|}{$\begin{array}{l}\text { Controls } \\
n=384,811\end{array}$}} & \multicolumn{2}{|l|}{ All IBD* } & \multicolumn{2}{|l|}{ UC } & \multicolumn{2}{|l|}{ CD } \\
\hline & & & $n=1,703$ & & $\mathrm{n}=706$ & & $\mathrm{n}=\mathbf{8 9 3}$ & \\
\hline & $\mathbf{n}$ & $\%$ & $\mathbf{n}$ & $\%$ & $\mathbf{n}$ & $\%$ & $\mathbf{n}$ & $\%$ \\
\hline \multicolumn{9}{|c|}{ Maternal age at childbirth, years } \\
\hline Mean (SD) & 29 & $(5.7)$ & 31 & $(4.9)$ & 32 & $(4.6)$ & 31 & $(5.1)$ \\
\hline \multicolumn{9}{|l|}{ Year of childbirth } \\
\hline 1990-1996 & 73,951 & 19.2 & 214 & 12.6 & 83 & 11.8 & 117 & 13.1 \\
\hline $1997-2003$ & 142,320 & 37.0 & 601 & 35.3 & 246 & 34.8 & 320 & 35.8 \\
\hline 2004-2010 & 168,540 & 43.8 & 888 & 52.1 & 377 & 53.4 & 456 & 51.1 \\
\hline \multicolumn{9}{|c|}{ Townsend deprivation index } \\
\hline 1 (Least deprived) & 92,359 & 24.0 & 511 & 30.0 & 225 & 31.9 & 250 & 28.0 \\
\hline 2 & 73,258 & 19.0 & 368 & 21.6 & 160 & 22.7 & 180 & 20.2 \\
\hline 3 & 74,083 & 19.3 & 311 & 18.3 & 145 & 20.5 & 149 & 16.7 \\
\hline 4 & 69,601 & 18.1 & 241 & 14.2 & 92 & 13.0 & 140 & 15.7 \\
\hline 5 (Most deprived) & 51,997 & 13.5 & 174 & 10.2 & 50 & 7.1 & 116 & 13.0 \\
\hline Missing & 23,513 & 6.1 & 98 & 5.8 & 34 & 4.8 & 58 & 6.5 \\
\hline \multicolumn{9}{|c|}{ Maternal smoking status } \\
\hline Non-smoking & 101,664 & 26.4 & 499 & 29.3 & 261 & 37.0 & 206 & 23.1 \\
\hline Ex-smoking & 127,528 & 33.1 & 618 & 36.3 & 276 & 39.1 & 306 & 34.3 \\
\hline Current smoking & 55,279 & 14.4 & 240 & 14.1 & 53 & 7.5 & 170 & 19.0 \\
\hline Missing & 100,340 & 26.1 & 346 & 20.3 & 116 & 16.4 & 211 & 23.6 \\
\hline
\end{tabular}


Table 2 Absolute risks of major congenital anomalies and system-specific congenital anomalies in children born to women with and without IBD before childbirth $(\mathrm{N}=386,514)$

\begin{tabular}{|c|c|c|c|c|c|c|c|c|}
\hline & \multirow{2}{*}{\multicolumn{2}{|c|}{$\begin{array}{l}\text { Controls } \\
n=384,811\end{array}$}} & \multirow{2}{*}{\multicolumn{2}{|c|}{$\begin{array}{l}\text { All IBD } \\
n=1,703\end{array}$}} & \multirow{2}{*}{\multicolumn{2}{|c|}{$\begin{array}{c}\text { UC } \\
n=706\end{array}$}} & \multirow{2}{*}{\multicolumn{2}{|c|}{$\begin{array}{c}\text { CD } \\
n=893\end{array}$}} \\
\hline & & & & & & & & \\
\hline & $\mathbf{n}$ & $n / 10,000$ & $\mathbf{n}$ & $n / 10,000$ & $\mathbf{n}$ & $n / 10,000$ & $\mathbf{n}$ & $n / 10,000$ \\
\hline Any major congenital anomalies & 10,645 & 277 & 46 & 270 & 26 & 368 & 17 & 190 \\
\hline Heart & 3,032 & 79 & 17 & 100 & 9 & 128 & 7 & 78 \\
\hline Limb & 2,059 & 54 & 5 & 29 & 4 & 57 & 1 & 11 \\
\hline Genital system & 1,542 & 40 & 8 & 47 & 4 & 57 & 2 & 22 \\
\hline Urinary system & 1,004 & 26 & 3 & 18 & 0 & - & 3 & 34 \\
\hline Chromosomal & 699 & 18 & 2 & 12 & 2 & 28 & 0 & - \\
\hline Oro-facial cleft & 529 & 14 & 3 & 18 & 1 & 14 & 2 & 22 \\
\hline Nervous system & 596 & 16 & 4 & 24 & 2 & 28 & 2 & 22 \\
\hline Musculoskeletal system & 515 & 13 & 2 & 12 & 2 & 28 & 0 & - \\
\hline Digestive system & 397 & 10 & 2 & 12 & 2 & 28 & 0 & - \\
\hline Eye & 388 & 10 & 0 & - & 0 & - & 0 & - \\
\hline Other malformations* & 375 & 10 & 0 & - & 0 & - & 0 & - \\
\hline Respiratory system & 253 & 7 & 2 & 12 & 1 & 14 & 1 & 11 \\
\hline Ear, face and neck & 103 & 3 & 1 & 6 & 1 & 14 & 0 & - \\
\hline Abdominal wall & 92 & 2 & 0 & - & 0 & - & 0 & - \\
\hline
\end{tabular}

$\mathrm{IBD}=$ Inflammatory bowel disease; $\mathrm{UC}=$ ulcerative colitis; $\mathrm{CD}=$ Crohn's disease

* e.g. asplenia, situs inversus, and skin disorders 
Table 3 Adjusted odds ratios for the association of maternal IBD with any major and system-specific congenital anomalies in children

\begin{tabular}{lllllll}
\hline & \multicolumn{2}{l}{ All IBD } & UC & \multicolumn{3}{l}{ CD } \\
\cline { 2 - 7 } & AOR $^{*}$ & $\mathbf{9 5 \% C l}$ & AOR $^{*}$ & $\mathbf{9 5 \% C l}$ & AOR $^{*}$ & $\mathbf{9 5 \% C l}$ \\
\hline Any major congenital anomaly & $\mathbf{0 . 9 8}$ & $\mathbf{0 . 7 3 - 1 . 3 1}$ & $\mathbf{1 . 3 4}$ & $\mathbf{0 . 9 1 - 1 . 9 7}$ & $\mathbf{0 . 6 8}$ & $\mathbf{0 . 4 1 - 1 . 1 3}$ \\
Heart & 1.24 & $0.75-2.05$ & 1.59 & $0.82-3.05$ & 0.97 & $0.42-2.24$ \\
Limb & 0.55 & $0.23-1.33$ & 1.05 & $0.39-2.81$ & 0.21 & $0.03-1.50$ \\
Genital system & 1.19 & $0.59-2.39$ & 1.43 & $0.53-3.84$ & 0.57 & $0.14-2.26$
\end{tabular}

${ }^{*}$ Odds ratios adjusted for maternal age in three categories, calendar year period of childbirth, Townsend Deprivation Index and maternal smoking status

OR=odds ratio; $95 \% \mathrm{Cl}=95 \%$ confidence interval 
Table 4 Numbers and proportions of 5-ASA, corticosteroids and Azathioprine/6-Mercaptopurine prescribed in the first trimester of pregnancy* for women with IBD

\begin{tabular}{lcrrrrrr}
\hline & \multicolumn{2}{l}{ All IBD } & \multicolumn{2}{l}{ UC } & \multicolumn{3}{l}{ CD } \\
\cline { 2 - 7 } & $\mathbf{n = 1 , 7 0 3}$ & $\mathbf{n = 7 0 6}$ & \multicolumn{2}{l}{$\mathbf{n = 8 9 3}$} \\
\cline { 2 - 7 } & $\mathbf{n}$ & $\%$ & $\mathbf{n}$ & $\%$ & $\mathbf{n}$ & $\%$ \\
\hline 5-ASA & 551 & 32.4 & 289 & 40.9 & 255 & 28.6 \\
Steroids & 209 & 12.3 & 98 & 13.9 & 108 & 12.1 \\
Azathioprine/6- & 149 & 8.7 & 34 & 4.8 & 115 & 12.9 \\
Mercaptopurine & & & & & & \\
\hline
\end{tabular}

*defined as from 4 weeks before to 12 weeks after conception

5-ASA=5-aminosalicylic acid; IBD=Inflammatory bowel disease; $U C=$ ulcerative colitis; $C D=C$ rohn's disease 
Table 5 Adjusted odds ratios for the risk of major congenital anomalies in children of women with IBD prescribed 5-ASA, steroid or azathioprine in the first trimester, compared with those of women with IBD not given that drug $(\mathrm{N}=1,703)$

\begin{tabular}{|c|c|c|c|}
\hline & \multicolumn{3}{|c|}{ Major congenital anomalies } \\
\hline & $\mathbf{n}$ & $n / 10,000$ & Adjusted OR* (95\%Cl) \\
\hline \multicolumn{4}{|l|}{ 5-ASA (n=551) } \\
\hline Any major congenital anomalies & 13 & 236 & $0.82(0.42-1.61)$ \\
\hline Heart & 4 & 73 & $0.66(0.18-2.48)$ \\
\hline Limb & 2 & 36 & $1.40(0.18-11.02)$ \\
\hline Genital system & 2 & 36 & $0.71(0.15-3.35)$ \\
\hline \multicolumn{4}{|l|}{ Corticosteroids (n=209) } \\
\hline Any major congenital anomalies & 3 & 144 & $0.48(0.15-1.50)$ \\
\hline Heart & 2 & 96 & $0.99(0.22-4.39)$ \\
\hline Limb & 0 & 0 & --- \\
\hline Genital system & 1 & 48 & $0.91(0.13-6.20)$ \\
\hline \multicolumn{4}{|l|}{$\begin{array}{l}\text { Azathioprine/6-Mercaptopurine }{ }^{\wedge} \\
(n=149)\end{array}$} \\
\hline Any major congenital anomalies & 5 & 336 & $1.27(0.48-3.39)$ \\
\hline Heart & 2 & 134 & $1.56(0.29-8.26)$ \\
\hline Limb & 0 & 0 & --- \\
\hline Genital system & 2 & 134 & 3.37 (0.64-17.73) \\
\hline
\end{tabular}




\section{Figure Legends}

Figure 1 Percentages of women prescribed Azathioprine/6-Mercaptopurine in the 15 months before pregnancy, during pregnancy and in the 15 months after childbirth in women with IBD $(N=1,162$ women registered with their GP throughout this period)

Figure 2 The changes in prescriptions of 5-aminosalicylic acid and Azathioprine/6-Mercaptopurine in women before pregnancy and in the first trimester and the number of flares later in pregnancy and postpartum. a) All IBD. b) Crohn's disease. c) UC 


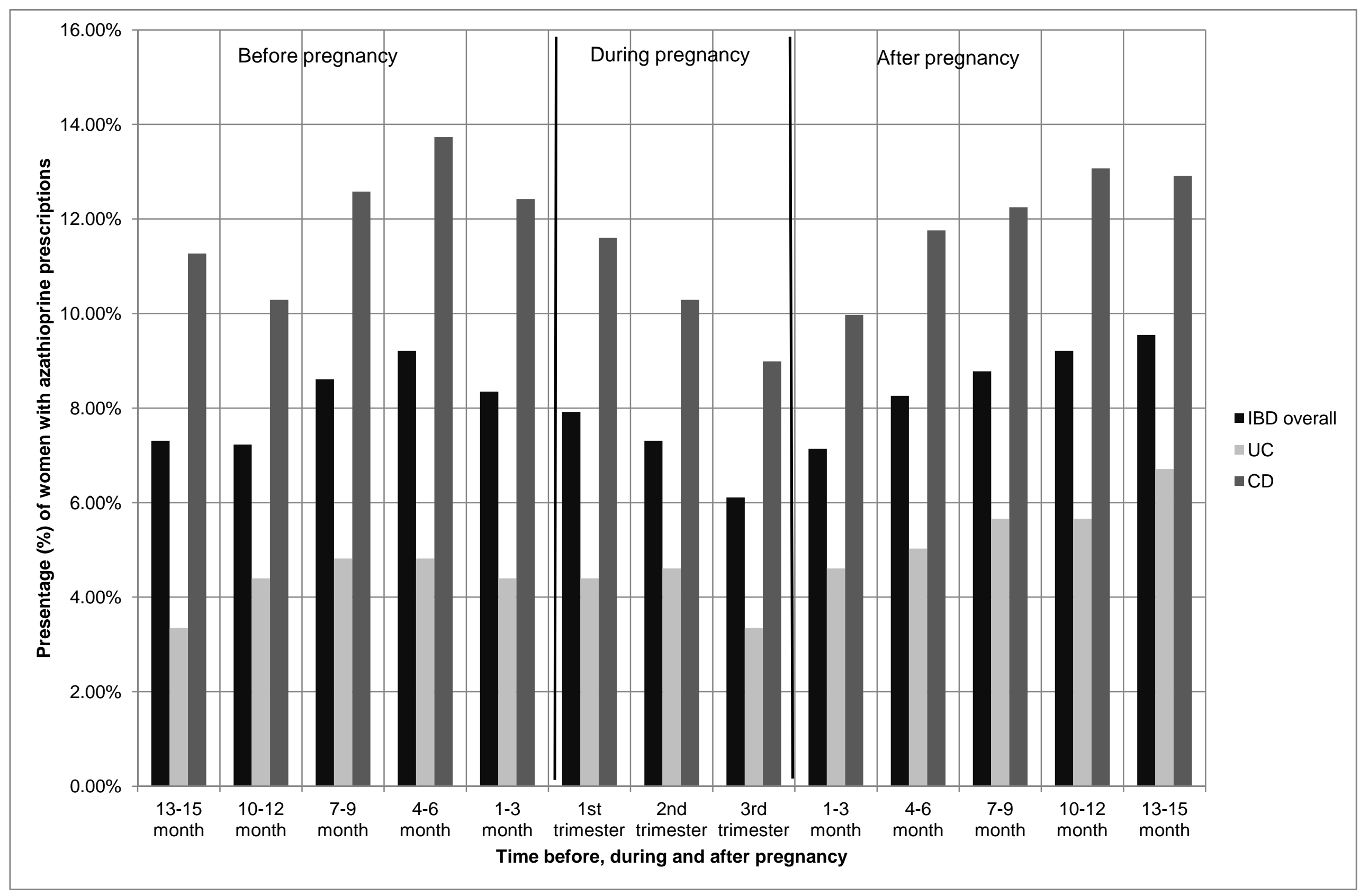


Figure 2a Prescriptions of 5-aminosalicylic acid and Azathioptine/6-Mercaptopurine in women with IBD before pregnancy and in the first trimester and the number of flares later in pregnancy and postpartum

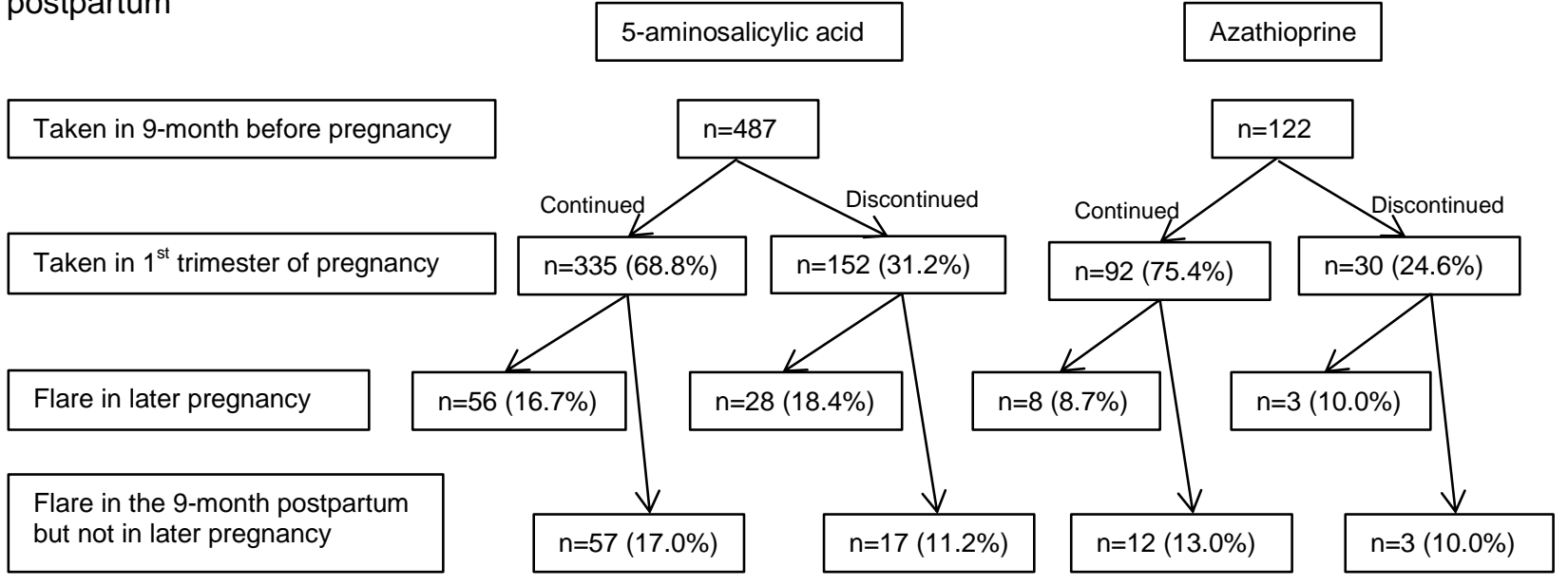

Figure 2b Prescriptions of 5-aminosalicylic acid and Azathioptine/6-Mercaptopurine in women with CD before pregnancy and in the first trimester and the number of flares later in pregnancy and postpartum

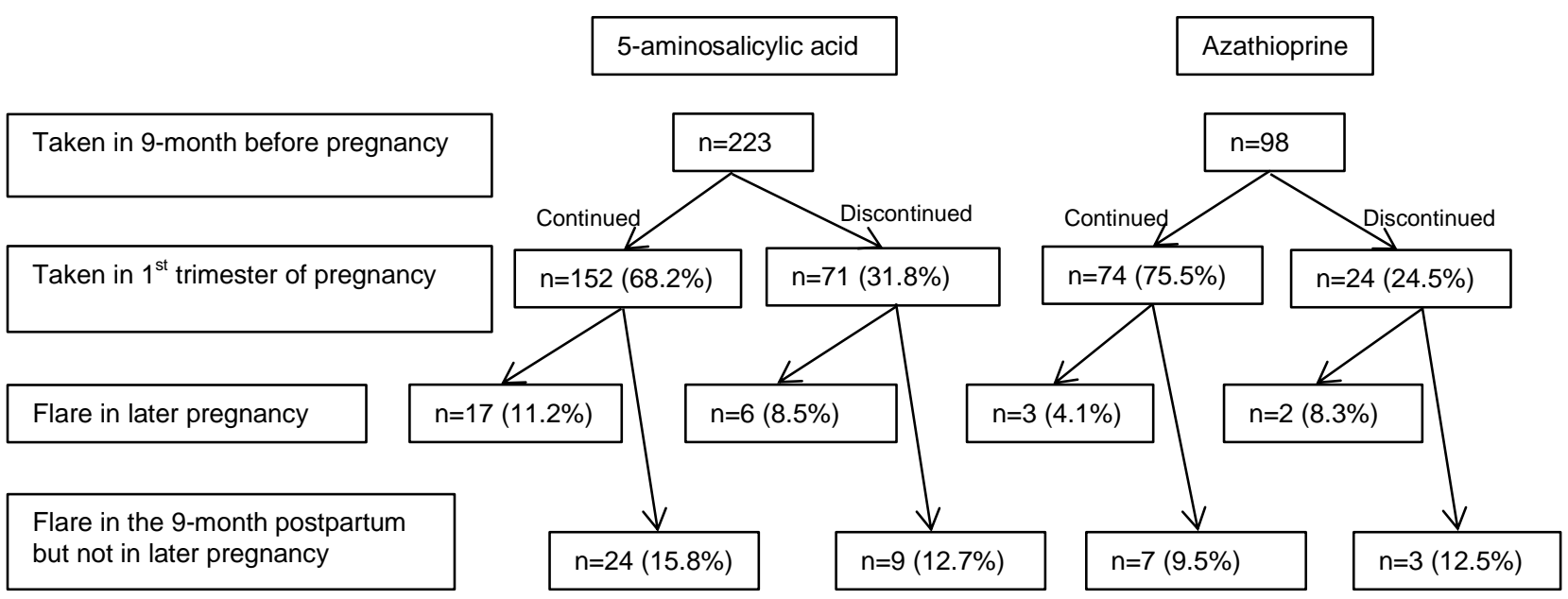

Figure 2c Prescriptions of 5-aminosalicylic acid and Azathioptine/6-Mercaptopurine in women with UC before pregnancy and in the first trimester and the number of flares later in pregnancy and postpartum

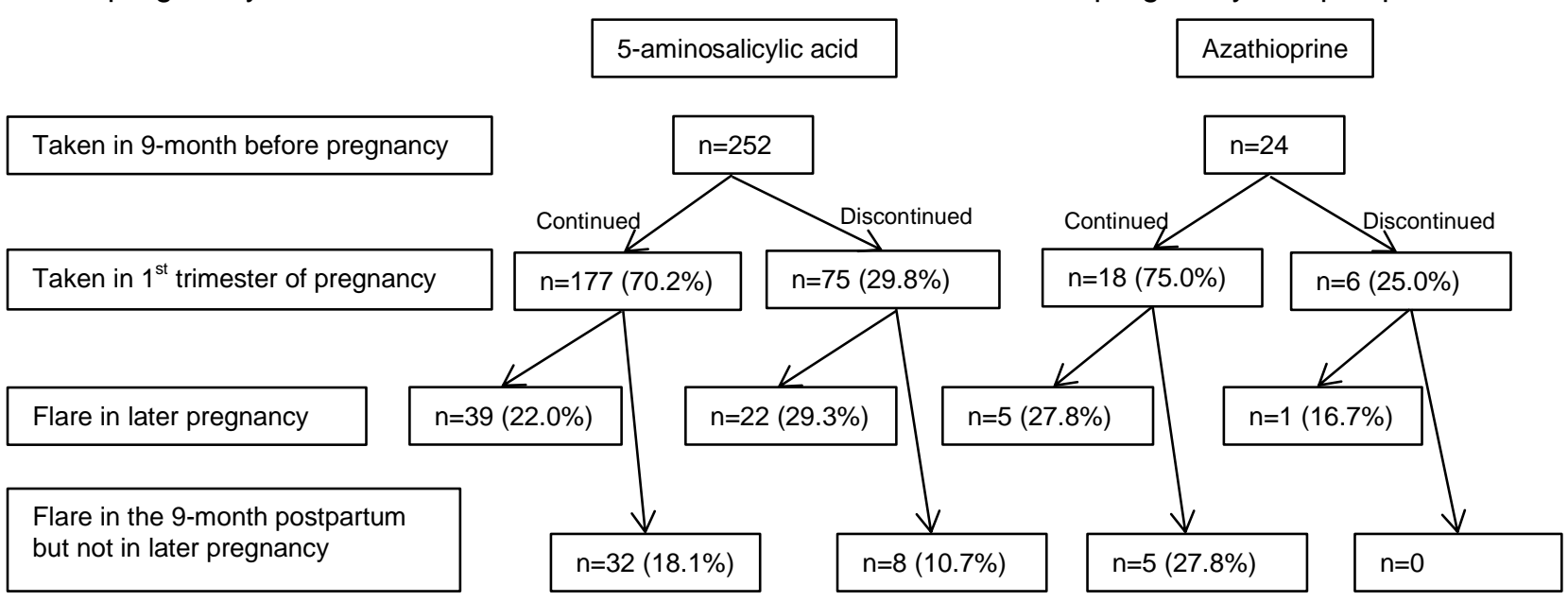

